consequence of this random inactivation is the variegation seen in the coats of female mice heterozygous for X-linked coat colour genes. The equal representation of wild and mutant phenotypes, and the presence of patches of each coat colour, suggest that at the time of decision in the early embryo, in each cell one $\mathrm{X}$ chromosome (chosen at random) remains active whereas any others are inactivated (in animals possessing several $\mathrm{X}$ chromosomes only one stays active). In the subsequent divisions of genetic development, the state of each homologue is faithfully reproduced; the adult cells descended from any particular embryonic cell after the time of decision therefore constitute a clone characterised by the presence of the same active $X$ chromosome (with the exception of the germ cells in which both $\mathrm{X}$ chromosomes remain active).

Two critical questions to ask about genetic development in eutherians are therefore: when does $\mathbf{X}$ activation take place; and what is the basis for its random nature? The closest estimate of the time of decision derives from the work of Gardner and Lyon (Nature, 231, 283; 1971) with chimaeric mice. Instead of the usual complete fusion between two embryos, they injected a cell taken from a blastocyst into another blastocyst. Because the donor and recipient carried different gene markers for coat colour, any cells in the adult coat descended from the injected embryonic cell can be distinguished. Since donor sex-linked markers showed

\section{BL Lac identified with giant galaxy}

THE peculiar object BL Lacertae seems to lie at the centre of a normal giant elliptical galaxy. This identification provides an estimate of the distance to BL Lac, and this in turn suggests that the central source is similar in spectral index and luminosity to QSOs such as $3 \mathrm{C} 48,3 \mathrm{C} 279$ and $3 \mathrm{C} 345$, provided those objects are at the distances indicated by a cosmological interpretation of their redshifts.

Those are the principal conclusions reached by Oke and Gunn as a result of a four year study of BL Lac (Astrophys. J. Lett., 189, L5; 1974). The observations were made with the multichannel spectrometer attached to the 200-inch telescope; some of the observations were made using a disk with diameter $5^{\prime \prime}$ and a surrounding ring with inner diameter $10^{\prime \prime}$ to study the extended regions of the source while obscuring light from the bright central feature, and these showed $\mathrm{H} \beta$ in absorption at a redshift of $z=0.07$.

Other data support this redshift estimate, and show that the galaxy is one of the most luminous known, with a magnitude of about -23 . Together with the growing evidence of similarities between $\mathrm{BL}$ Lac and QSOs this identification is one of the key advances of recent years. Far from being a blueshifted QSO, as had seemed possible, BL Lac can now be fitted into the cosmological picture of receding QSOs quite satisfactorily. The distance to the source is some $350 \mathrm{Mpc}$, and this raises the same questions about its energy generation mechanisms that apply for very remote QSOs. The energy requirements are extreme, but as Oke and Gunn say "It is interesting, however, that the same phenomena at approximately the same level of outrageousness exist for BL Lac as for higher-redshift QSOs if they are placed at the distances suggested by their redshifts".

J. G. variegation in the heterozygous condition, $\mathrm{X}$ activation must take place after the blastocyst stage (otherwise the donor cell would have suffered previous $\mathrm{X}$ activation and its descendents would all be of one phenotype in the adult).

By taking advantage of a mutant which influences the random nature of $\mathrm{X}$ activation, Ohno and his colleagues (Cell, 1, 175; 1974) have recently developed an ingenious approach to estimate the number of cells in the embryonic mouse at the time of decision. Their experiments rely on the concept that the clonal composition of embryos immediately after $\mathrm{X}$ activation can be expressed as the binomial $(p+q)^{n}$, where $n$ is the number of cells and $p$ and $q$ are the probabilities of activation of each chromosome, both equal to 0.5 in the wild type animal. In other words, given a population of animals it is possible to predict the frequencies with which any particular clonal composition will be generated. Unfortunately, this formula cannot be directly extrapolated to the adult animal, because distortions in the initial clonal composition must inevitably take place as the embryo grows; for only some, and not necessarily a random selection, of the embryonic cells will be utilised during formation of each adult clone. The formula which determines the clonal composition of an adult phenotype must therefore be written as $\left(p^{\prime}+q^{\prime}\right)^{n^{\prime}}$, where $n^{\prime}$ is the number of progenitor cells from which the adult clone is descended, and $p^{\prime}$ and $q^{\prime}$ have values corresponding to the relative frequencies of each active $X$ chromosome in the set of progenitor cellsand this, of course, in turn depends upon the clonal composition of the embryo from which the progenitor cells are descended.

Because these changes in cell composition make it impossible to determine $n$ directly from the adult phenotypes, Ohno et al. have used a special situation to allow this analysis. In one particular condition only, changes in cell composition cannot occur during development; this is the case when all the copies of one $\mathrm{X}$ chromosome (either the maternal or paternal) were by chance activated. This generates a monoclonal embryo, which can in turn give rise only to monoclonal adult. But these two extreme cases each occur with a very low frequency $\left(0.5^{n}\right)$; as Ohno et al. point out, this creates the paradox that, even given a value for $n$ as low as 20 , such fractions would occur with frequencies too low determine by experiment in any researcher's lifetime (of the order of one in a million mice). To achieve a more manageable situation, they therefore utilised a mutation of the $\mathrm{X}$ chromosome $\left(0^{\text {hv }}\right)$, which they have previously characterised, and which causes preferential activation of the $\mathrm{X}$ chromosome carrying it in heterozygotes where the other chromosome is wild type (Nature new Biol., 245, 92; 1973; Cell, 1, 3; 1974). The $0^{\text {hv }}$ mutation seems to be an extreme version of alleles at the Cattanach's "controlling element" locus which maps in the middle of the mouse $\mathrm{X}$-linkage group; and earlier analyses of chromosomes carrying this allele have suggested that the mechanism of decision is for one $\mathrm{X}$ chromosome to be activated whereas any others in the cell are inactivated (reviewed by Brown and Chandra, Proc. natn. Acad. Sci. U.S.A., 70, 195; 1973).

When the $O^{h v} \mathrm{X}$ chromosome carries the mutant gene $B l o$ and the other $\mathbf{X}$ chromosome carries the corresponding wild type allele, regions of the coat of the mice are light coloured if derived from cells in which the $0^{h v}$ Blo $\mathrm{X}$ chromosome was activated, and are dark if derived from cells in which the wild type $\mathrm{X}$ chromosome was activated. Because of the preferential activation of the $0^{h v} \mathrm{X}$ chromosome, the frequency of occurrence of mice with coats largely of Blo phenotype is appreciable. To obtain values for the occurence of two, rather than just one, of the possible clonal compositions, in addition to assessing the proportion of mice with coats derived entirely from Blo cells, Ohno et al. also determine the frequency with which mice displayed coats almost entirely of Blo phenotype, but with just one dark spot (corresponding to one coat progenitor cell in which the wild 